\title{
Transverse spectrum of bremsstrahlung in finite condensed media
}

\author{
X. Feal ${ }^{*}$ and R. A. Vazquez \\ Departamento de Física de Partículas \& Instituto Galego de Física de Altas Enerxías \\ Universidade de Santiago de Compostela, 15782 Santiago, Spain
}

(Received 8 October 2018; published 2 January 2019)

\begin{abstract}
A formalism is presented in which the radiation of photons off high energy electrons during a multiple scattering process with finite condensed media can be evaluated for a general interaction. We show that the arising Landau-Pomeranchuk-Migdal suppression for finite size targets saturates at some characteristic photon energy. Medium coherence effects in the photon dispersion relation can be also considered leading to a dielectric suppression or transition radiation effects in the soft part of the spectrum. The main results of our formulation are presented for a Debye screened interaction and its well-known FokkerPlanck approximation, showing that for finite size targets or for the angular distributions of the final particles the differences between both scenarios cannot be reconciled into a single redefinition of the medium transport parameter $(\hat{q})$. Our predictions are in very good agreement with the experimental data collected at SLAC.
\end{abstract}

DOI: $10.1103 /$ PhysRevD.99.016002

\section{INTRODUCTION}

The Landau-Pomeranchuk-Migdal (LPM) suppression is a well-known effect that has been extensively studied. Interference phenomena in a multiple scattering scenario was initially considered by Ter-Mikaelian as the mechanism regulating the amount of scattering centers which can coherently emit as a single bremsstrahlung source [1]. A classical evaluation of this effect for a semi-infinite medium was soon introduced by Landau and Pomeranchuk $[2,3]$ and later completed by Migdal [4] for the quantum case by means of a Boltzmann transport equation for the electron. This calculation has shown [5] that except for the spin corrections for hard photons, the LPM suppression for an averaged target still agrees with the expected classical behavior of the infrared divergence. Further and more recent developments in various approaches have been introduced since then by Blankenbecler and Drell [6-8], Zakharov [9-15], the Baier-Dokshitzer-Mueller-SchiffPeigné group (BDMPS) [16], Baier and Katkov [17-19], and Wiedemann and Gyulassy [20], and extensive reviews were presented in $[21,22]$. We note, however, that all the existing calculations were done in the Fokker-Planck approximation, which both in the Boltzmann transport

\footnotetext{
*xabier.feal@igfae.usc.es

†vazquez@igfae.usc.es
}

Published by the American Physical Society under the terms of the Creative Commons Attribution 4.0 International license. Further distribution of this work must maintain attribution to the author(s) and the published article's title, journal citation, and DOI. Funded by SCOAP. approach $[4,16]$ and in the path integral formulation $[9,20]$ lead to a Gaussian distribution of momenta. In this approximation, then, the transport properties of the medium have to be adequate to take into account the neglected large momentum tails of the original Debye screened or Coulomb interactions. Few works, on the other hand, considered the finite target case, which has always been problematic and sometimes misunderstood lacking a general formulation. Also, the angular distribution of the final particles has not been studied in general [20]. Taking into account these remarks, no result has ever been given beyond the FokkerPlanck approximation, that also accounts for the transverse photon and electron spectrum, and which includes in a natural way the finiteness of the target.

In this paper, we have developed a formalism and a Monte Carlo code which allows for the computation of the bremsstrahlung spectrum of finite targets, arbitrary interactions and with a full control of the kinematics of the process, so that specific cuts on momenta of the final electron and photon can be applied. In Sec. II A we will briefly explain the LPM effect in a classical scenario and then we will review the formalism of the quantum case by considering finite lengths, general interactions and the angular distribution of the final particles. In Sec. II B we will make connection with the already existing works by showing that for semi-infinite mediums within the FokkerPlanck approximation the result of Migdal [4] and the equivalent rederivations by Bell [5] and Zakharov [10] are exactly reproduced in our formalism. In Sec. III, we will present and compare our results with the experimental data of SLAC [23]. Finally, we end in Sec. IV with some conclusions. 


\section{FORMALISM AND CALCULATION}

\section{A. Finite length and general interactions}

In this section we will introduce the general formalism applicable to finite targets and general interactions.

It has been predicted by Ter-Mikaelian [1] and Landau and Pomeranchuk [2] that at high energies the BetheHeitler cross section [24] stops being applicable to extended media. In order to understand this phenomenon we start with the emission amplitude for a process consisting in a collision with $(n)$ sources

$\mathcal{M}_{\mathrm{em}}^{(n)}=-i e \int d^{4} y \bar{\Psi}_{f}^{(n)}(y) \gamma^{\mu} A_{\mu}^{\lambda}(y) \Psi_{i}^{(n)}(y)+\mathcal{O}\left(e^{2}\right)$,

where in the Coulomb gauge $A_{\mu}^{\lambda}(y)=\mathcal{N}(k) \epsilon_{\mu}^{\lambda} e^{i k \cdot y}$ is a free photon of momentum $k$, polarization $\epsilon^{\lambda}=\left(0, \epsilon^{\lambda}\right)$ and normalization $\mathcal{N}(k)=\sqrt{2 \pi / \omega}, \Psi_{i, f}^{(n)}(x)$ the incoming and outgoing electron wave functions under the external field of the medium and $e=\sqrt{\alpha}$ the electron charge. Since in the $\omega \rightarrow 0$ limit the number of photons diverges, in virtue of the soft photon theorem [25], the classical approximation holds [26] and we can replace

$J_{k}(x)=\bar{\Psi}_{f}^{(n)}(x) \gamma_{k} \Psi_{i}^{(n)}(x) \rightarrow J_{k}(x) \equiv v_{k}(t) \delta^{3}(\boldsymbol{x}-\boldsymbol{x}(t))$,

where $\boldsymbol{v}(t) \equiv \dot{\boldsymbol{x}}(t)$ is the electron velocity. Using the completeness relation

$$
\sum_{\lambda} \epsilon_{i}^{\lambda} \epsilon_{k}^{\lambda}=\delta_{i k}-\frac{k^{i} k^{k}}{\omega^{2}}
$$

we can find a correspondence with a classical amplitude $[5,27]$

$\mathcal{M}_{\mathrm{em}}^{(n)}=-i e \mathcal{N}(k) \int_{-\infty}^{+\infty} d t\left(\frac{\boldsymbol{k}}{\omega} \times \boldsymbol{v}(t)\right) e^{i \omega t-i \boldsymbol{k} \cdot \boldsymbol{x}(t)}$,

One can consider the integration over time as the point in which the photon is emitted. This observation becomes manifest by letting the electron describe a discretized trajectory, with velocities $\boldsymbol{v}_{j}$ for $j=1, \ldots, n_{c}+1$ and piecewise path $\boldsymbol{x}_{j}=\boldsymbol{x}_{j-1}+\boldsymbol{v}_{j-1}\left(t_{j}-t_{j-1}\right)$, where $n_{c}$ is the number of collisions. Equation (4) then produces

$$
\mathcal{M}_{\mathrm{em}}^{(n)}=e \mathcal{N}(k) \frac{1}{\omega} \sum_{j=1}^{n_{c}} \boldsymbol{\delta}_{j} e^{i \varphi_{j}},
$$

where we find a superposition of $n_{c}$ single Bethe-Heitler like amplitudes [24] of the form

$$
\boldsymbol{\delta}_{j} \equiv \boldsymbol{k} \times\left(\frac{\boldsymbol{v}_{j+1}}{\omega-\boldsymbol{k} \cdot \boldsymbol{v}_{j+1}}-\frac{\boldsymbol{v}_{j}}{\omega-\boldsymbol{k} \cdot \boldsymbol{v}_{j}}\right),
$$

interfering with a phase $\varphi_{j} \equiv \omega t_{j}-\boldsymbol{k} \cdot \boldsymbol{x}_{j}$. The evaluation of the square of (5) leads to a total emission intensity between the photon solid angle $\Omega_{k}$ and $\Omega_{k}+d \Omega_{k}$ given by

$\omega \frac{d I}{d \omega d \Omega_{k}}=\frac{e^{2}}{(2 \pi)^{2}}\left(\sum_{j=1}^{n_{c}} \boldsymbol{\delta}_{j}^{2}+2 \operatorname{Re} \sum_{j=1}^{n_{c}} \sum_{i=1}^{j-1} \boldsymbol{\delta}_{j} \cdot \boldsymbol{\delta}_{i} e^{i \varphi_{i}^{j}}\right)$,

where we have split the sum in a diagonal and a nondiagonal contribution. The interfering behavior of the above sum is governed by the phase change between two arbitrary collisions or emission elements

$$
\begin{aligned}
\varphi_{i}^{j} & \equiv \varphi_{j}-\varphi_{i}=k_{\mu}\left(x_{j}^{\mu}-x_{i}^{\mu}\right)=\int_{z_{i}}^{z_{j}} d z \frac{k_{\mu} p^{\mu}(z)}{p_{0}} \\
& \simeq \omega(1-\beta) \int_{z_{i}}^{z_{j}} d z+\omega \int_{z_{i}}^{z_{j}} d z \frac{\delta \boldsymbol{p}^{2}(z)}{2 \beta p_{0}^{2}}
\end{aligned}
$$

where $p_{0}$ is the initial electron energy, $\delta \boldsymbol{p}(z)$ is the accumulated momentum change of the electron at $z$ with respect to the photon direction and $\beta=|\boldsymbol{v}|=\sqrt{1-m_{e}^{2} / p_{0}^{2}}$ the electron velocity. This phase can be made maximal for large emission angles and/or photon frequencies, provided that $\varphi_{i}^{i+1} \gg 1$ for any two consecutive collisions. In that case the nondiagonal sum in (7) cancels and we are left with a totally incoherent superposition of $\left(n_{c}\right)$ single BetheHeitler intensities, with a maximal intensity of

$$
\omega \frac{d I_{\text {sup }}}{d \omega d \Omega_{k}}=\frac{e^{2}}{(2 \pi)^{2}} \sum_{j=1}^{n_{c}} \boldsymbol{\delta}_{j}^{2}
$$

In this regime emission decouples and all the scatterings can be considered to be independently emitting. In the opposite case, when the emission angle and/or photon energy are small so that the phase vanishes, the internal structure of the scattering is irrelevant. This observation becomes manifest by setting $\varphi_{j}=0$ in (5), so we are left with the first and last terms only and intensity acquires the minimum value

$$
\omega \frac{d I_{\mathrm{inf}}}{d \omega d \Omega_{k}}=\frac{e^{2}}{(2 \pi)^{2}}\left|\sum_{j=1}^{n_{c}} \boldsymbol{\delta}_{j}\right|^{2},
$$

which can be interpreted as a Bethe-Heitler intensity with a final velocity $\boldsymbol{v}_{n_{c}+1}$ due to the coherent deflection with all the medium centers. In this regime the entire medium acts as a single independent emission element. This behavior is a consequence of the well known soft photon theorem $[25,28]$, although in the LPM literature it is known as the Ternovskii-Shul'ga-Fomin emission [29,30]. The suppression from the superior (incoherent) plateau of radiation (9) to the inferior (coherent) plateau (10) is known as the LPM effect for mediums of arbitrary size. Notice that in the 
infinite medium limit $\left(n_{c} \gg 1\right)$ the coherent plateau can be neglected, since the soft photon theorem is not observed and then the suppression is infinite.

The above classical arguments can be made quantitative and hold also for a quantum evaluation of the amplitude. By Fourier transforming electron states $\Psi_{i, f}(x)$ to the momentum space, we can write for the quantum amplitude (1)

$$
\begin{aligned}
\mathcal{M}_{\mathrm{em}}^{(n)}= & e \mathcal{N}(k) \int \frac{d^{3} \boldsymbol{p}(z)}{(2 \pi)^{3}} \int_{0}^{l} d z \exp \left(i \frac{k_{\mu} p^{\mu}(z)}{p_{0}}\right) \frac{d}{d z} \\
& \times\left\{S_{s_{n} s}^{\mathrm{el}}(p(l), p(z) ; l, z) \frac{f_{s s^{\prime}}^{\lambda}(z)}{k_{\mu} p^{\mu}(z)}\right. \\
& \left.\times S_{s^{\prime} s_{0}}^{\mathrm{el}}(p(z)+k, p(0) ; z, 0)\right\},
\end{aligned}
$$

where we used the shorthand notation

$f_{s s^{\prime}}^{\lambda}(z) \equiv \epsilon_{\mu}^{\lambda}(k) p_{0} \sqrt{\frac{m_{e}}{p_{0}-\omega}} \bar{u}_{s}(p) \gamma^{\mu} u_{s^{\prime}}(p+k) \sqrt{\frac{m_{e}}{p_{0}}}$.

Here $S_{s_{2} s_{1}}^{\mathrm{el}}\left(p_{2}, p_{1} ; l_{2}, l_{1}\right)$ stands for the beyond eikonal evaluation of the elastic amplitudes for an electron to go from momentum $p_{1}$ to $p_{2}$ and from spin $s_{1}$ to $s_{2}$ due the amount of matter between $l_{1}$ and $l_{2}$, thus given by

$S_{s_{n} s_{0}}^{(n)}\left(p_{n}, p_{0} ; z_{n}, z_{0}\right) \equiv\left(\prod_{k=1}^{n-1} \int \frac{d^{3} \boldsymbol{p}_{k}}{(2 \pi)^{3}}\right)\left(\prod_{i=1}^{n} S_{s_{i} s_{i-1}}^{n\left(z_{i}\right)}\left(q_{i}\right)\right)$,

where we discretized the medium and thus $q_{i} \equiv p_{i}-p_{i-1}$ is the 4-momentum transfer at the layer $(i)$ of $n\left(z_{i}\right)$ scattering centers, and sum over intermediate spins is assumed. At high energies the amplitude for a change of momentum $q$ at the $i$ th layer verifies [31]

$$
\begin{aligned}
S_{s_{i} s_{i-1}}^{n\left(z_{i}\right)}(q)= & 2 \pi \delta\left(q^{0}\right) \delta_{s_{i-1}}^{s_{i}} \beta e^{-i q_{\|} z_{i}} \\
& \times \int d^{2} \boldsymbol{y}_{t} e^{-i \boldsymbol{q}_{\boldsymbol{t}} \cdot \boldsymbol{y}} \exp \left[-i \frac{g}{\beta} \sum_{k=1}^{n\left(z_{i}\right)} \chi\left(\boldsymbol{y}_{t}-\boldsymbol{r}_{t}^{k}\right)\right],
\end{aligned}
$$

where $y_{t}$ is an impact parameter, and $\boldsymbol{r}^{k}=\left(\boldsymbol{r}_{t}^{k}, z_{k}\right)$ is the position of the scattering center at the layer $k$. The external field characterizing the medium is given by $(n)$ single Debye static sources with screening $\mu_{d} \simeq \alpha m_{e} Z^{1 / 3}$, coupled with strength $g=Z \alpha$ to the electron, of the form

$\chi_{0}^{(1)}(\boldsymbol{x}) \equiv \int_{-\infty}^{+\infty} d s A_{0}^{(1)}(\boldsymbol{x}), \quad A_{0}^{(1)}(\boldsymbol{x})=\frac{Z \alpha}{|\boldsymbol{x}|} e^{-\mu_{d}|\boldsymbol{x}|}$.

The amplitude (11), which corresponds to a sum of the single emission elements shown in Fig. 1, can be squared

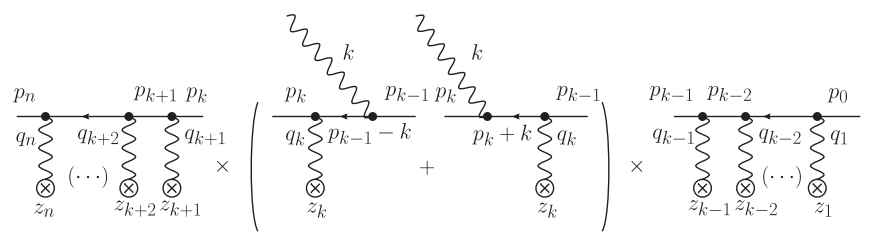

FIG. 1. Diagrammatic representation of the single emission elements appearing in a discretization in the variable $z$, of the amplitude $\mathcal{M}_{\mathrm{em}}^{(n)}$ given at Eq. (11).

and averaged over medium configurations of infinite transverse size $R \rightarrow \infty$ in a finite length $l$, summed over final states, and averaged over initial states, leading to an intensity of emission in the photon solid angle $\Omega_{k}$ and $\Omega_{k}+d \Omega_{k}$ and per unit of medium transverse size and unit time of

$$
\begin{aligned}
\omega \frac{d I}{d \omega d \Omega_{k}}= & \left(\frac{e}{2 \pi}\right)^{2}\left(\prod_{i=1}^{n} \int \frac{d^{3} \boldsymbol{p}_{i}}{(2 \pi)^{3}}\right)\left(\prod_{k=1}^{n} \phi\left(\delta \boldsymbol{p}_{k}, \delta z\right)\right) \\
& \times\left(h^{n}(y)\left|\sum_{j=1}^{n} \boldsymbol{\delta}_{j}^{n} e^{i \varphi_{0}^{j}}\right|^{2}+h^{s}(y)\left|\sum_{j=1}^{n} \delta_{j}^{s} e^{i \varphi_{0}^{j}}\right|^{2}\right),
\end{aligned}
$$

where the spin nonflip currents $\boldsymbol{\delta}_{j}^{n}$ are given by (6) and the spin flip currents are given by

$$
\delta_{j}^{s} \equiv \frac{1}{1-\beta_{k} \hat{\boldsymbol{k}} \cdot \boldsymbol{v}_{j+1}}-\frac{1}{1-\beta_{k} \hat{\boldsymbol{k}} \cdot \boldsymbol{v}_{j}} .
$$

Here we have introduced explicitly the possibility of a medium with a refractive index, by using $\beta_{k}$, the photon velocity. The functions $h^{n}(y)$ and $h^{s}(y)$ are the diagonal and nondiagonal sum in spins and helicities of the squared emission vertex (12), given by

$$
h^{n}(y)=\frac{1}{2}\left(1+(1-y)^{2}\right), \quad h^{s}(y)=\frac{1}{2} y^{2},
$$

and $y=\omega / p_{0}$ is the fraction of energy carried by the photon. They produce two contributions of the same order, the last one, however, only noticeable when $y \approx 1$ due to $h^{s}(y)$. In what follows we will neglect this contribution by assuming that $y \ll 1$. Within the same approximation we will assume also that the electron 4-momentum change in the emission vertex is negligible and $\beta=1$ unless otherwise required. The local elastic weights arising in the averaging of the square of (13) are given by

$$
\begin{aligned}
\phi(\delta \boldsymbol{p}, \delta z)= & \exp \left(-n_{0}(z) \delta z \sigma_{t}^{(1)}\right)(2 \pi)^{3} \delta^{3}(\delta \boldsymbol{p}) \\
& +2 \pi \delta\left(\delta p_{0}\right) \beta \Sigma_{2}(\delta \boldsymbol{p}, \delta z),
\end{aligned}
$$

where we can define the no collision probability $\exp \left(-n(z) \delta z \sigma_{t}^{(1)}\right)$ in the layer of length $\delta z$ and density 
$n_{0}(z)$ times the forward distribution $\delta^{3}(\delta \boldsymbol{p})$, and the collisional distribution $\Sigma_{2}(\boldsymbol{q}, \delta z)$ after an incoherent scattering with the centers in $\delta z$

$$
\begin{aligned}
\Sigma_{2}(\boldsymbol{q}, \delta z) \equiv & \int d^{2} \boldsymbol{x} e^{-i \boldsymbol{q} \cdot \boldsymbol{x}} \exp \left(-n_{0}(z) \delta z \sigma_{t}^{(1)}\right) \\
& \times\left(\exp \left(n_{0}(z) \delta z \sigma(\boldsymbol{x})\right)-1\right),
\end{aligned}
$$

which satisfies a Moliere's equation with boundary condition $\Sigma_{2}(\boldsymbol{q}, 0)=0$. The required single elastic cross sections at (19) and (20) can be shown to satisfy $\sigma_{t}^{(1)} \equiv$ $\sigma(\mathbf{0})$ where, at leading order in $Z \alpha$ using (15)

$$
\sigma(\boldsymbol{x}) \equiv \frac{4 \pi(Z \alpha)^{2}}{\beta^{2} \mu_{d}^{2}} \mu_{d}|\boldsymbol{x}| K_{1}\left(\mu_{d}|\boldsymbol{x}|\right)+\mathcal{O}(Z \alpha)^{3}
$$

The expression (16) is our main result. It can be directly used for numerical evaluation for arbitrary medium lengths or medium properties. In addition, it can be shown that in the continuum limit it can be cast as a combination of six path integrals [32]. These path integrals reduce to the well known Migdal's or Zakharov results in the appropriate limits. In addition to a numerical evaluation of (16), we will derive also an heuristic formula for finite size targets to qualitatively understand the interference phenomena. The coherence length defined by the phase modulates the amount of scatterers which can be considered a single and independent unit of emission in the squared amplitude. We then define the length $\delta l=z_{j}-z_{i}$ in which the phase becomes larger than unity, which using (8) becomes

$$
\varphi_{i}^{j} \simeq \frac{\omega}{2 p_{0}^{2}}\left(m_{e}^{2} \delta l+\hat{q}(\delta l)^{2}\right) \equiv 1,
$$

then we get

$$
\delta l(\omega) \equiv \frac{m_{e}^{2}}{2 \hat{q}}\left(\sqrt{1+\frac{8 \hat{q} p_{0}^{2}}{m_{e}^{4} \omega}}-1\right) .
$$

We also define the frequency $\omega_{c}$ at which the coherence length becomes equal to $l$, the medium total length, thus $\omega_{c} \simeq p_{0}^{2} /\left(m_{e}^{2} l+\hat{q} l^{2}\right)$, and the frequency $\omega_{s}$ in which the coherence length becomes equal to a mean free path $\lambda$, $\omega_{s} \simeq p_{0}^{2} /\left(m_{e}^{2} \lambda+\hat{q} l \lambda / 2\right)$. Since we assume the medium to be finite we further impose to (23) $\delta l(\omega)=l$ for $\omega>\omega_{c}$. In the coherence length $\delta l(\omega)$ the internal scattering structure is irrelevant since the phase can be neglected, and the centers in $\delta l(\omega)$ act coherently like a single scattering source with the total equivalent charge in $\delta l(\omega)$. Since in the entire medium $l$ there are $l / \delta l(\omega)$ coherence lengths, then we write the incoherent sum

$$
\begin{aligned}
\omega \frac{d I}{d \omega}(l)= & \frac{l}{\delta l(\omega)} e^{2} \int \frac{d \Omega_{k}}{(2 \pi)^{2}} \int \frac{d^{3} \delta \boldsymbol{p}}{(2 \pi)^{3}} \\
& \times\left(h^{n}(y)\left|\boldsymbol{\delta}_{1}^{n}\right|^{2}+h^{s}(y)\left|\delta_{1}^{s}\right|^{2}\right) \phi(\delta \boldsymbol{p}, \delta l(\omega)) .
\end{aligned}
$$

By integrating in the photon solid angle $\Omega_{k}$ and using (19), we find

$\omega \frac{d I(l)}{d \omega}=\frac{l}{\delta l(\omega)} \frac{e^{2}}{\pi^{2}} \int_{0}^{\pi} d \theta \sin (\theta) F(\theta) \Sigma_{2}(\delta \boldsymbol{p}, \delta l(\omega))$,

where the electron momentum change is $|\delta \boldsymbol{p}|=$ $2 p_{0} \beta \sin (\theta / 2)$ and

$$
\begin{aligned}
F(\theta)= & {\left[\frac{1-\beta^{2} \cos \theta}{2 \beta \sin (\theta / 2) \sqrt{1-\beta^{2} \cos ^{2}(\theta / 2)}}\right.} \\
& \left.\times \log \left[\frac{\sqrt{1-\beta^{2} \cos ^{2}(\theta / 2)}+\beta \sin (\theta / 2)}{\sqrt{1-\beta^{2} \cos ^{2}(\theta / 2)}-\beta \sin (\theta / 2)}\right]-1\right] .
\end{aligned}
$$

This last integral (25) can be numerically evaluated and the resulting intensity is exact for $\omega \gg \omega_{s}$ and $\omega \ll \omega_{c}$. A simple interpolation formula in the Fokker-Planck approximation can be obtained from (25) by integrating its two asymptotic values, i.e., $\delta l(\omega) \gtrsim l$ and $\delta l(\omega) \lesssim \lambda$, and then interpolating both regions. One finds

$\omega \frac{d I(l)}{d \omega}=\frac{l}{\delta l(\omega)} \frac{2 e^{2}}{\pi} \frac{1+n_{m}(\omega)}{3 A+n_{m}(\omega)} \log \left(1+A n_{m}(\omega)\right)$,

where $n_{m}(\omega) \simeq 2 \hat{q} \delta l(\omega) / m_{e}^{2}$ is a measure of the accumulated transverse momentum in a coherence length and $A=e^{-(1+\gamma)}$, with $\gamma$ Euler's constant.

The general behavior of these results can be summarized in Fig. 2 where the photon intensity is pictured as a function of the photon frequency. Above the saturation frequency $\omega_{s}$ the photon resolves all the internal structure of the scattering, the medium emits as a total incoherent sum of $n_{c}=l / \lambda$ Bethe-Heitler intensities, where $n_{c}$ is the average number of collisions. In this regime, the photon intensity scales with $l$. Notice that total suppression can occur provided $\omega_{s}$ becomes larger than $p_{0}$, which causes that electrons with energies larger than $p_{0}^{l p m}=m_{e}^{2} / n_{0} \sigma_{t}^{(1)}$ experiment the bremsstrahlung suppression in all their spectrum. For smaller frequencies the number of independent emitters, using (23), decreases with $\sqrt{\omega}$ whereas the charge of each element logarithmically grows with $\log (1 / \sqrt{\omega})$. This suppression stops at $\omega_{c}$, where the coherence length (23) acquires the maximum value $l$, the medium emits as a single entity and intensity saturates to Weinberg's soft photon theorem [25]. The presence of a medium modifies 


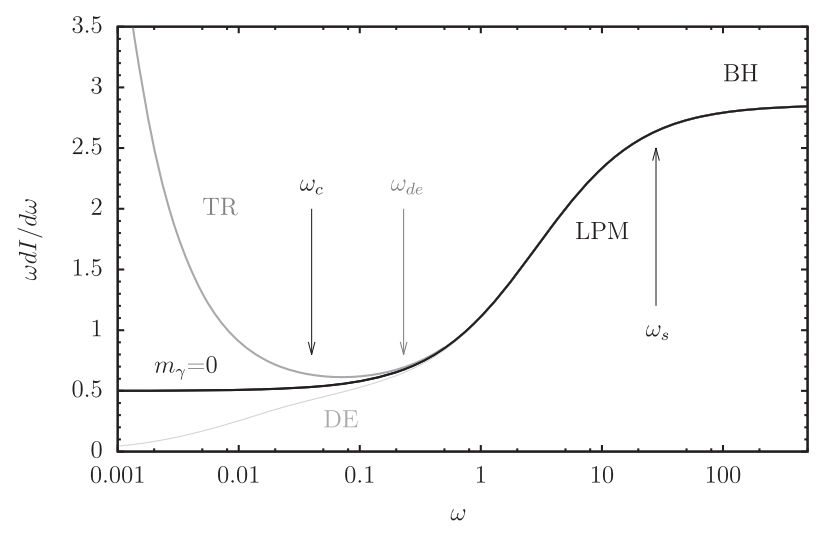

FIG. 2. Schematic representation of the bremsstrahlung regimes for several scenarios. Totally incoherent Bethe-Heitler superposition $(\mathrm{BH})$, Landau-Pomeranchuk-Migdal suppression (LPM), totally coherent Bethe-Heitler superposition $\left(m_{\gamma}=0\right)$, dielectric suppression (DE) and transition radiation (TR). See text for definition of the characteristics frequencies $\omega_{c}, \omega_{s}, \omega_{\mathrm{de}}$.

the photon dispersion relation and substantially changes this picture in the soft limit. For the energies considered here the photon has velocity given by

$$
\beta^{2}(k)=1-\frac{\omega_{p}^{2}}{\omega^{2}}
$$

where $\omega_{p}^{2} \simeq 4 \pi Z \alpha n_{0} / m_{e} \equiv m_{\gamma}^{2}$ is the plasma frequency, which can be interpreted as a photon mass $m_{\gamma}$. This scenario induces an additional source of suppression due to the fact that the wavelength of a photon of frequency $\omega$ is now larger than in the vacuum case and thus

$$
k_{\mu} p^{\mu}\left(\omega_{p}\right) \simeq k_{\mu} p^{\mu}(0)+\frac{m_{\gamma}^{2}}{2 \omega} .
$$

This extra term further suppresses the coherent plateau at $\omega<\omega_{\mathrm{de}}$, where $k_{\mu} p^{\mu}(0) \equiv m_{\gamma}^{2} / 2 \omega_{\mathrm{de}}$, i.e., $\omega_{\mathrm{de}}^{2}=\omega_{p}^{2} l \omega_{c}$, since the denominators of (6) and (17), defined by (29), grow for smaller frequencies. This suppression is called the dielectric effect and holds for a totally homogeneous space or infinite medium. However, if the electron passes through vacuum to a medium and then again to vacuum, or in general through structured targets where density cannot be considered constant, then the definition (29) becomes local for each photon emission point. The photon emitted at the last leg (the vacuum) then satisfies $m_{\gamma}=0$, whereas the first leg photon satisfies $m_{\gamma} \neq 0$, thus it can be shown that an interference destroys the dielectric suppression in the coherence plateau, dramatically enhancing the intensity for $\omega<\omega_{\mathrm{de}}$. This is called transition radiation $[27,33]$. Both of these effects have been implemented in our formalism and Monte Carlo. In Fig. 2 we show qualitatively the dielectric effect and the transition radiation together with their characteristic frequency $\omega_{\mathrm{de}}$.

\section{B. Semi-infinite length and Fokker-Planck approximation}

In Migdal's calculation of the intensity [4] the angular distribution of photons is not considered, the medium is assumed to be semi-infinite $l \rightarrow \infty$ and the Moliere's equation satisfied by the elastic distributions is replaced by the Fokker-Planck equation. As a result, the electron distributions of momenta, which have a Yukawa form for a Debye screened interaction (15), are replaced by Gaussian distributions and the large $p_{t}$ tails are neglected. We will briefly explain here how within these approximations Eq. (16) leads to Migdal's result [4] and also the equivalent rederivations by Bell [5] and Zakharov [10].

We assume from here onwards a constant density $n_{0}(z) \equiv n_{0}$ so from (19) the electron mean free path is constant and reads $\lambda \equiv 1 / n_{0} \sigma_{t}^{(1)}$. In this elastic propagation the electron acquires a squared momentum transfer additive with the traveled length $l$. Indeed, from (19) we find

$$
\frac{\partial}{\partial l}\left\langle\delta \boldsymbol{p}^{2}(l)\right\rangle=n_{0} \sigma_{t}^{(1)}\left\langle\delta \boldsymbol{p}^{2}(\delta l)\right\rangle \equiv 2 \hat{q},
$$

where we defined the transport parameter $\hat{q}$. Using (30) we can write then $\hat{q}=(\eta / 2) \times n_{0} \sigma_{t}^{(1)} \mu_{d}^{2}$, where $\eta$ is a parameter to be determined a posteriori. Then the Fokker-Planck approximation for (20) is obtained as

$$
n_{0} l \sigma(\boldsymbol{x}) \simeq n_{0} l \sigma(\mathbf{0})-\frac{1}{2} \hat{q} l \boldsymbol{x}^{2}
$$

so that (20) acquires a Gaussian form

$$
\Sigma_{2}^{G}(\boldsymbol{q}, \delta z) \equiv \frac{2 \pi}{\hat{q} \delta z} \exp \left(-\frac{\boldsymbol{q}^{2}}{2 \hat{q} \delta z}\right)
$$

Inserting (32) in (19) the integration in the internal momenta at (16) is now straightforward. It can be shown [32] that after taking the continuous limit $\delta z \rightarrow 0$, integrating in the final photon momentum and taking the $l \rightarrow \infty$ limit, the intensity of photons for $y \ll 1$ is given by

$$
\begin{aligned}
\omega \frac{d I(l)}{d \omega}= & l \frac{2 e^{2}}{\pi} \sqrt{\frac{\hat{q} \omega}{2 p_{0}^{2}}} \int_{0}^{\infty} d z \exp \left(-\frac{z}{\sqrt{2} s}\right) \\
& \times\left(\sin \left(\frac{z}{\sqrt{2} s}\right)+\cos \left(\frac{z}{\sqrt{2} s}\right)\right) \\
& \times\left(\frac{1}{z^{2}}-\frac{1}{\sinh ^{2}(z)}\right),
\end{aligned}
$$

which agrees with Migdal/Bell/Zakharov predictions [4,5,10]. In Migdal's calculations, the parameter $s$ is defined recursively, in terms of an implicit expression 
which is related to the definition of $\hat{q}$. Here, we define $s \equiv\left(2 p_{0} / m_{e}^{2}\right) \sqrt{\hat{q} / \omega}$ and introduce the parameter $\eta$ to account for the freedom in the definition of $\hat{q}$.

An estimation of the value of $\eta$ can be given by considering the momentum transfer in a single collision $\delta l \lesssim \lambda$ which is given, using (19), by
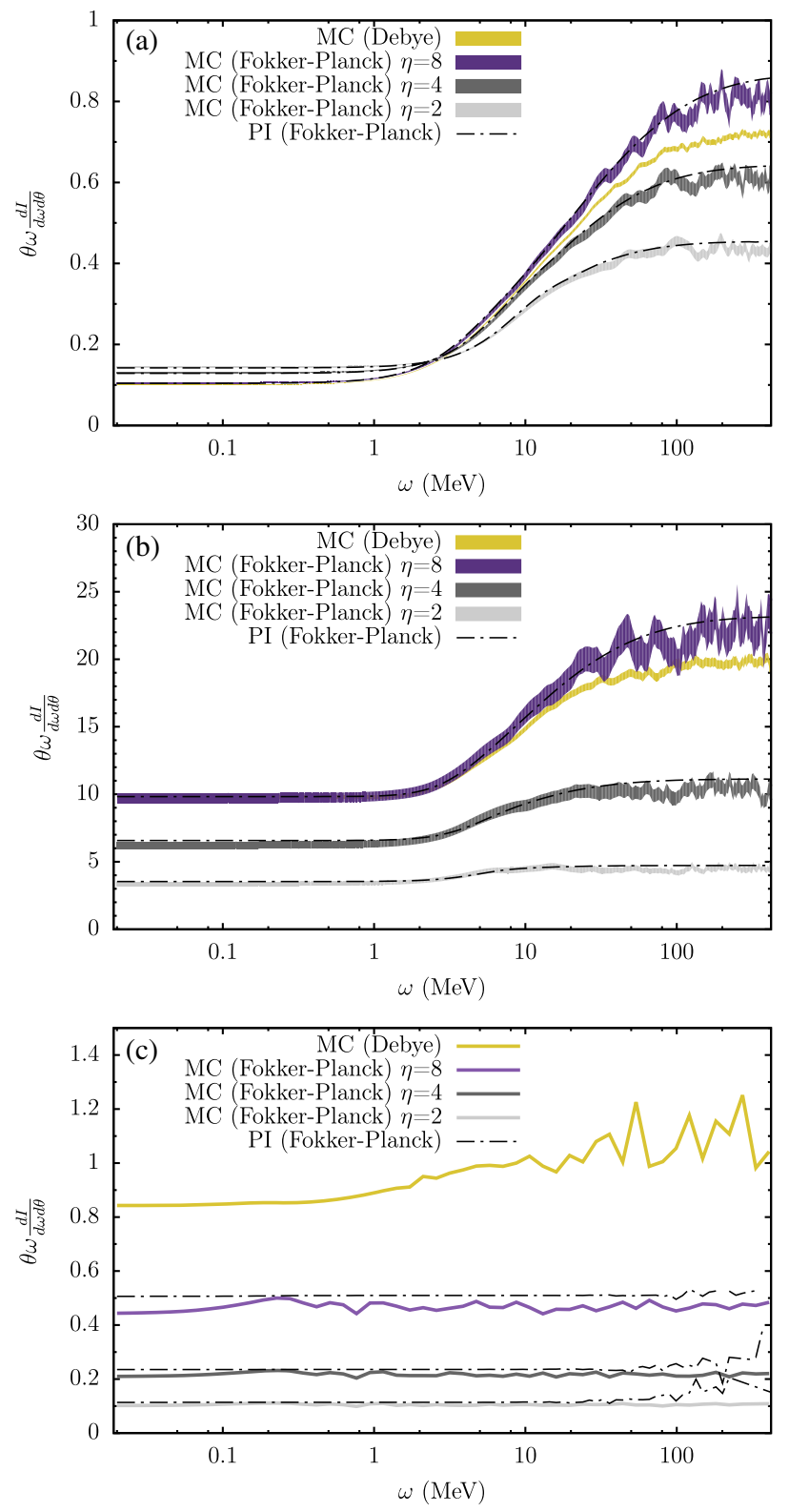

FIG. 3. Differential spectrum of photons emitted by an electron of $p_{0}=8 \mathrm{GeV}$ traversing a sheet of gold of $l=0.023 \mathrm{~mm}$ for photon angles $\theta_{k}=0.01 / \gamma(\mathrm{a}), \theta_{k}=2 / \gamma(\mathrm{b})$ and $\theta_{k}=10 / \gamma(\mathrm{c})$, where $\gamma \equiv p_{0} / m_{e}$, in the Monte Carlo (MC) evaluations of (16) in the Debye interaction (yellow), in the Fokker-Planck approximation with $\eta=8$ (purple), $\eta=4$ (dark grey), and $\eta=2$ (light grey), together with the corresponding path integral (PI) limits of (16) in the Fokker-Planck approximation (dot-dashed lines). Bands show the statistical uncertainty of the Monte Carlo.

$$
\left\langle\delta \boldsymbol{p}^{2}(\delta l)\right\rangle=\mu_{d}^{2}\left(2 \log \left(\frac{2 p_{0}}{\mu_{d}}\right)-1\right)=\mu_{d}^{2} \eta
$$

where the correction $\eta$ to $\mu_{d}^{2}$ takes into account the long tail of the Debye interaction (15) and a maximum momentum transfer of $|\delta \boldsymbol{p}|=2 p_{0}$ is allowed in a single collision. High momentum changes are suppressed at high energies, however, due to the functions (6) and (17) in (16). We have checked that a maximum momentum transfer of $|\delta \boldsymbol{p}| \simeq 2.5 m_{e}$ matches the single emission and then we write for $\eta$ in (34) instead

$\eta=\left(2 \log \left(\frac{2.5 m_{e}}{\mu_{d}}\right)-1\right)=\left(2 \log \left(\frac{2.5}{\alpha Z^{1 / 3}}\right)-1\right)$.

This effective momentum transfer under bremsstrahlung agrees with Bethe's [24] estimation $\eta=2 \log \left(183 / Z^{1 / 3}\right)$ within less than $3 \%$ deviation in the range $Z=(1,100)$. The above relations hold, however, for the single scattering regime $\delta z \leq \delta l$, so they can be used only to fix $\eta$ and thus $\hat{q}$ in the incoherent plateau. For the coherent plateau a
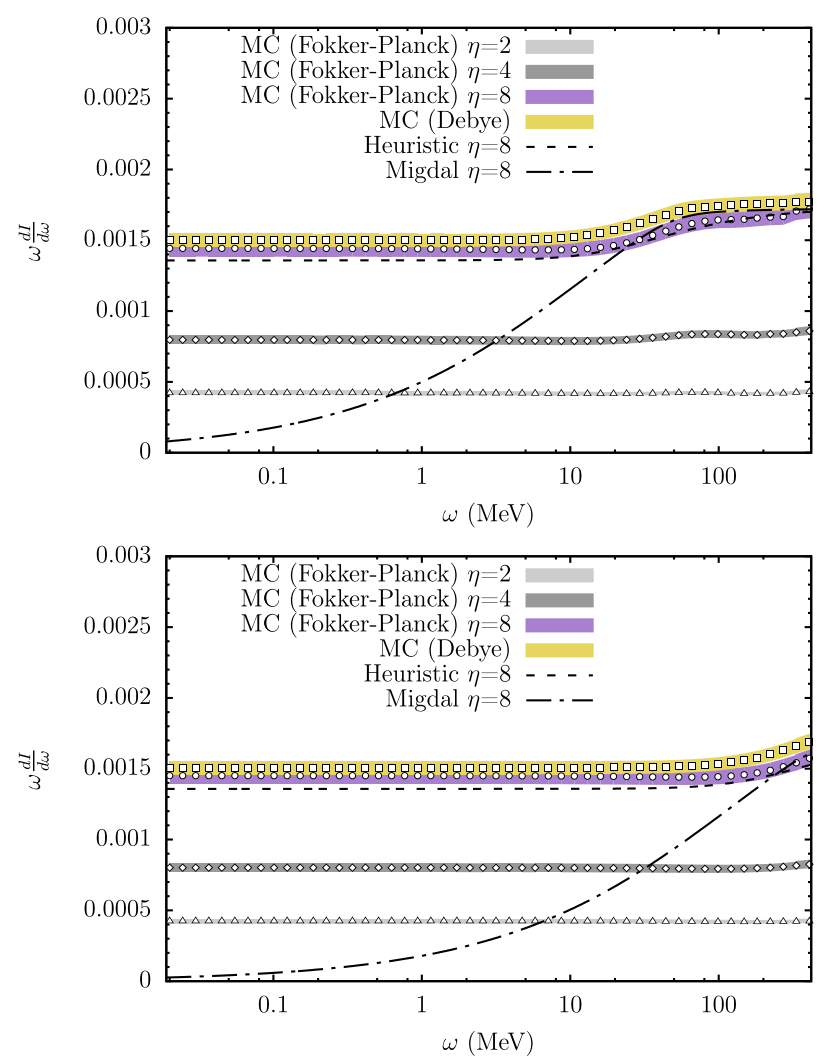

FIG. 4. Intensity of photons emitted by an electron of $p_{0}=$ $8 \mathrm{GeV}$ (top) and $p_{0}=25 \mathrm{GeV}$ (bottom) after traversing a sheet of gold of length $l=0.0038 \mathrm{~mm}$ in the Monte Carlo (MC) evaluation of (16) in the Debye interaction (squares), the FokkerPlanck approximation with $\eta=8$ (circles), $\eta=4$ (diamonds), and $\eta=2$ (triangles). Also shown our heuristic formula (25) and the Migdal prediction (33). 
medium-length dependent fit for $\eta$ has to be employed. Correspondingly, a single Fokker-Planck approximation can not fit both the upper and lower ends of the bremsstrahlung spectrum unless the medium length is very large, in which case the lower plateau occurs at very low frequencies and can be neglected. In our numerical calculations, we will choose the value of $\eta$ to match the Debye calculations in the incoherent plateau.

\section{RESULTS}

Expression (16) can be numerically evaluated for arbitrary interaction models, for finite size and arbitrarily structured targets with dielectric suppression and transition radiation effects included. We have developed a Monte Carlo code to evaluate this intensity by means of discretized paths with a typical step of $\delta z=0.1 \lambda$. In a typical run we computed $10^{4}$ paths for 50 frequencies and 100 photon angles, spanning from $\sim 10^{3}$ steps for the shortest medium to $\sim 10^{5}$ steps for the largest. In order to check that our results are correct we implemented also the Fokker-Planck approximation (32) for (19) in this
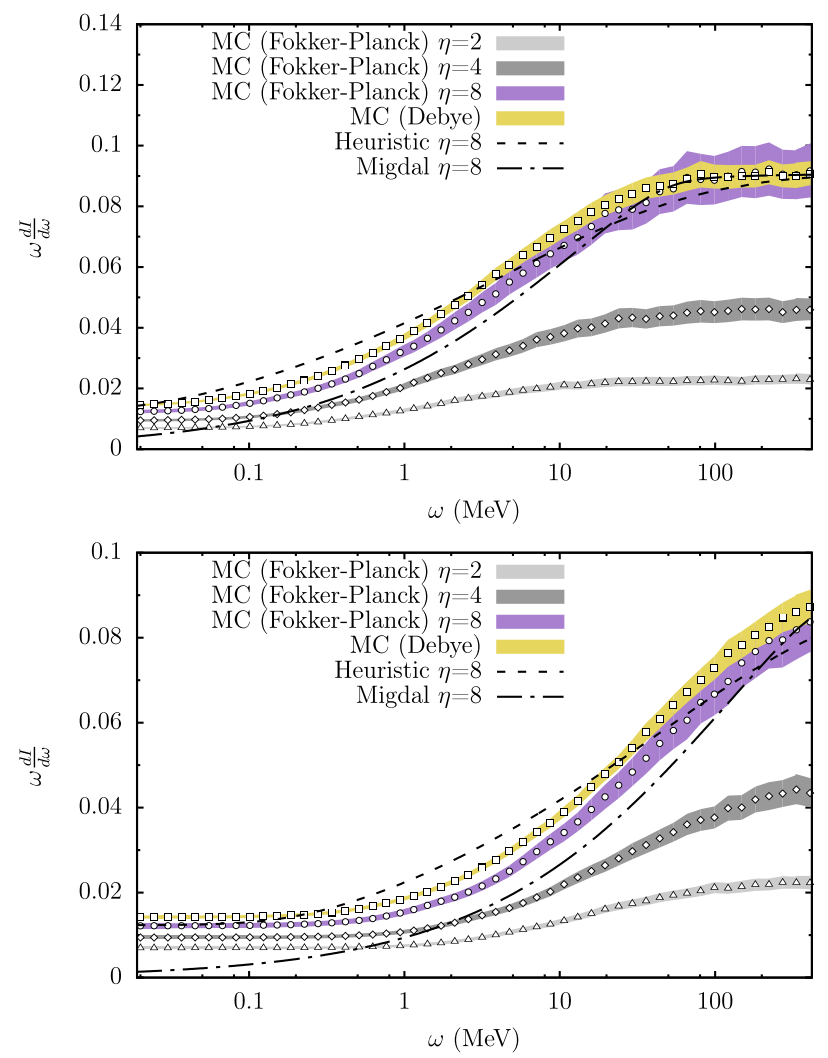

FIG. 5. Intensity of photons emitted by an electron of $p_{0}=$ $8 \mathrm{GeV}$ (top) and $p_{0}=25 \mathrm{GeV}$ (bottom) after traversing a sheet of gold of length $l=0.2 \mathrm{~mm}$ in the Monte Carlo (MC) evaluation of (16) in the Debye interaction (squares), the Fokker-Planck approximation with $\eta=8$ (circles), $\eta=4$ (diamonds) and $\eta=2$ (triangles). Also shown our formula (25) and the Migdal prediction (33). discretized approach and compared with the $\delta z \rightarrow 0$ limit of (16), which within this approximation produces six integrable Gaussian path integrals extending the Boltzmann transport approach [4] to finite mediums [20]. We present our result for the Debye and Fokker-Planck cases for targets of lengths $l=0.0038,0.023$ and $0.2 \mathrm{~mm}$, corresponding to an average of $n_{c}=142,862$, and 7502 collisions, respectively, for electrons of $p_{0}=8$ and $25 \mathrm{GeV}$, in order to compare to the SLAC data presented in $[23,34,35]$. A systematic study and comparison with other experimental results will be presented elsewhere [32]. For gold we obtain an estimate for the Debye mass of $\mu_{d}=16 \mathrm{keV}$, a transport parameter of $\hat{q}=(\eta / 2) \times 1.89 \mathrm{keV}^{3}$, and fix the effective parameter $\eta=8$. The plasma frequency is $\omega_{p}=0.080 \mathrm{keV}$ (see also [36]). In Fig. 3 we show the differential photon intensity as a function of the photon energy for various fixed emission angles for an electron of $p_{0}=8 \mathrm{GeV}$ traversing a gold sheet of $l=0.023 \mathrm{~mm}$. The path integral limit is also shown, and an excellent agreement with the Fokker-Planck Monte Carlo evaluation is found. At low angles, the Fokker-Planck approximation overestimates the intensity by $\sim 20 \%$. However, at larger angles the
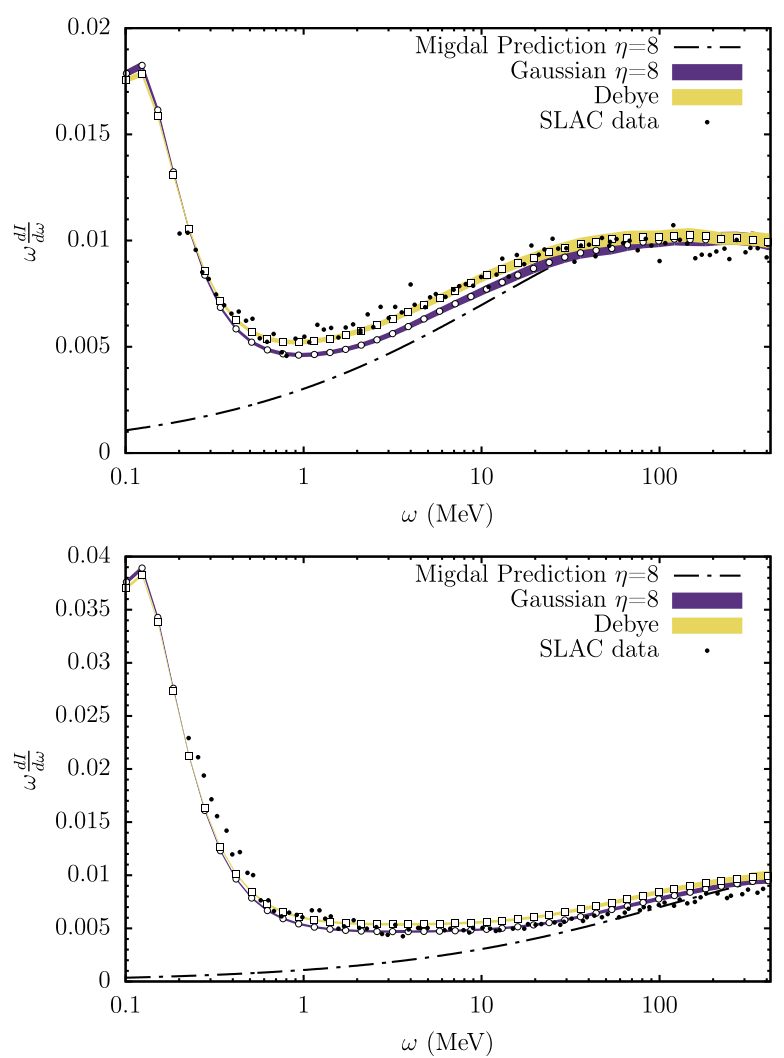

FIG. 6. Intensity of photons emitted by an electron of $p_{0}=$ $8 \mathrm{GeV}$ (top) and $p_{0}=25 \mathrm{GeV}$ (bottom) after traversing a sheet of gold of length $l=0.023 \mathrm{~mm}$ in the Debye interaction (open squares) and the Fokker-Planck approximation with $\eta=8$ (circles) with the dielectric and transition radiation effect included, compared to SLAC experimental data [23] (solid circles) rescaled. 


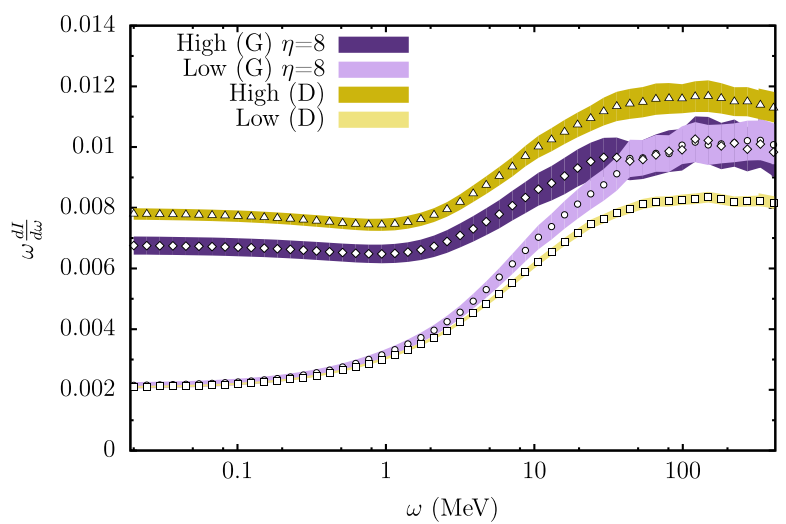

FIG. 7. Intensity of photons emitted by an electron of $p_{0}=$ $8 \mathrm{GeV}$ after traversing a sheet of gold of length $l=0.023 \mathrm{~mm}$ from electrons with final transverse momentum $\boldsymbol{p}_{t}>4 \mathrm{MeV}$ in the Debye interaction (triangles) and the Fokker-Planck approximation with $\eta=8$ (diamonds), and with $\boldsymbol{p}_{t}<4 \mathrm{MeV}$ in the Debye interaction (squares) and the Fokker-Planck approximation with $\eta=8$ (circles).

Fokker-Planck approximation underestimates the intensity, in particular only half of the real emission is obtained for $\theta=10 \gamma^{-1}$. In Fig. 4 we show the angular integrated spectrum for a sheet of gold of $l=0.0038 \mathrm{~mm}$ for electron energies of $p_{0}=8$ and $25 \mathrm{GeV}$. We see that the parameter $\eta=8$ in the Fokker-Planck approximation matches the incoherent plateau but mismatches the coherent plateau. Also shown are the expression (25) and the Migdal prediction (33) both in the Fokker-Planck approximation. The predicted characteristic frequencies are $\omega_{c}=8 \mathrm{MeV}$ and $\omega_{s}=1.1 \mathrm{GeV}$ for $p_{0}=8 \mathrm{GeV}$, and $\omega_{c}=80 \mathrm{MeV}$ and $\omega_{s}=11 \mathrm{GeV}$ for $p_{0}=25 \mathrm{GeV}$, being in good agreement with the obtained Monte Carlo results.

In Fig. 5 we show the same results for a sheet of gold of $l=0.2 \mathrm{~mm}$. We see that Migdal prediction becomes a good approximation for $n_{c} \geq 10^{4}$, i.e., when the coherent plateau can be neglected. For this length we predicted $\omega_{c}=8 \mathrm{keV}$ and $\omega_{s}=60 \mathrm{MeV}$ for $p_{0}=8 \mathrm{GeV}$, and $\omega_{c}=80 \mathrm{keV}$ and $\omega_{s}=588 \mathrm{MeV}$ for $p_{0}=25 \mathrm{GeV}$. All these values are in well agreement with the Monte Carlo evaluation.

In Fig. 6 we show the dielectric and transition radiation effect implementation both in the Debye interaction and the Fokker-Planck approximation, and compare with SLAC data [23], for a sheet of gold of $l=0.023 \mathrm{~mm}$ and electron energies of $p_{0}=8$ and $25 \mathrm{GeV}$. The characteristic frequency predictions $\omega_{\mathrm{de}}=0.6 \mathrm{MeV}$ for $p_{0}=8 \mathrm{GeV}$ and $\omega_{\mathrm{de}}=1.9 \mathrm{MeV}$ for $p_{0}=25 \mathrm{GeV}$ and the comparison with experimental data are in very good agreement. The LPM characteristic frequencies are given in this case by $\omega_{c}=0.48 \mathrm{MeV}$ and $\omega_{s}=418 \mathrm{MeV}$ for $p_{0}=8 \mathrm{GeV}$, and $\omega_{c}=4.7 \mathrm{MeV}$ and $\omega_{s}=4 \mathrm{GeV}$ for $p_{0}=25 \mathrm{GeV}$.

In Fig. 7 we show the intensity of bremsstrahlung for electron with a final transverse momentum $|\delta \boldsymbol{p}|<4 \mathrm{MeV}$ or $|\delta \boldsymbol{p}|>4 \mathrm{MeV}$. We see that the Fokker-Planck results do not reproduce well the Debye calculation. Although large differences could be expected for the case of $|\delta \boldsymbol{p}|>4 \mathrm{MeV}$, as the Fokker-Planck approximation underestimate the long tails of the transverse momentum distribution, it is perhaps more surprising to find that also cutting at low $p_{t}$ produces different results: the FokkerPlanck result overestimates the emission in this case at large frequencies.

\section{CONCLUSIONS}

A formalism implemented with a Monte Carlo method has been presented which is able to evaluate the bremsstrahlung intensity in a multiple scattering scenario under a general interaction. We have also found an heuristic formula which describes the LPM effect for finite size targets. Dielectric and transition radiation effects related to effective photon masses in the medium dispersion relation are included in this formalism if needed. Our results reproduce the experimental data of SLAC.

We have shown that the Fokker-Planck approximation does not fit well the differential angular spectrum, especially if kinematical cuts are applied in the final particles. In the integrated spectrum, the Fokker-Planck approximation fails to reproduce the spectrum. If the $\hat{q}$ is fixed using the incoherent plateau, then the coherent plateau is not well reproduced, unless a length dependent definition of the transport properties of the medium is used.

\section{ACKNOWLEDGMENTS}

We thank J. Alvarez-Muñiz, N. Armesto, C. Salgado, and J. Sanchez-Guillen for reading the manuscript and for discussions. We thank the grant María de Maeztu Unit of Excellence of Spain and the support of Xunta de Galicia under the Project No. ED431C2017. This paper has been partially done under the Project No. FPA2017-83814-P of Ministerio de Ciencia, Innovación y Universidades (Spain). 
[1] M. L. Ter-Mikaelian, The interference emission of highenergy electrons, Zh. Eksp. Teor. Fiz. 25, 296 (1953).

[2] L. Landau and I. Pomeranchuk, The limits of applicability of the theory of bremsstrahlung by electrons and of the creation of pairs at large energies, Dokl. Akad. Nauk Ser. Fiz. 92, 535 (1953).

[3] L. Landau, Electron-cascade processes at ultra-high energies, Dokl. Akad. Nauk Ser. Fiz. 92, 735 (1953).

[4] A. B. Migdal, Bremsstrahlung and pair production in condensed media at high energies, Phys. Rev. 103, 1811 (1956).

[5] J. S. Bell, Bremsstrahlung from multiple scattering, Nucl. Phys. 8, 613 (1958).

[6] R. Blankenbecler and S.D. Drell, Quantum treatment of beamstrahlung, Phys. Rev. D 36, 277 (1987).

[7] R. Blankenbecler, Multiple scattering and functional integrals, Phys. Rev. D 55, 2441 (1997).

[8] R. Blankenbecler, Structured targets and the Landau Pomeranchuk Migdal effect, Phys. Rev. D 55, 190 (1997).

[9] B. G. Zakharov, Landau Pomeranchuk Migdal effect for finite size targets, Zh. Eksp. Teor. Fiz. 64, 737 (1996).

[10] B. G. Zakharov, Fully quantum treatment of the LandauPomeranchuk-Migdal effect in QED and QCD, JETP Lett. 63, 952 (1996).

[11] B. G. Zakharov, Light cone path integral approach to the Landau Pomeranchuk Migdal effect, Yad. Fiz. 61, 924 (1998).

[12] B. G. Zakharov, Radiative energy loss of high energy quarks in finite size nuclear matter and quark gluon plasma, JETP Lett. 65, 615 (1997).

[13] B. G. Zakharov, Light cone path integral approach to the LPM effect and the SLAC data on bremsstrahlung from high energy electrons, Yad. Fiz. 62, 1075 (1999).

[14] B. G. Zakharov, Fully quantum treatment of the Landau Pomeranchuk Migdal effect in QED and QCD, JETP Lett. 63, 952 (1996).

[15] B. G. Zakharov, Transverse spectra of induced radiation, JETP Lett. 70, 176 (1999).

[16] R. Baier, Yu. L. Dokshitzer, A. H. Mueller, S. Peigné, and D. Schiff, The Landau-Pomeranchuk-Migdal effect in QED, Nucl. Phys. B478, 577 (1996).

[17] V. N. Baier and V. M. Katkov, The theory of the Landau Pomeranchuk Migdal effect, Phys. Rev. D 57, 3146 (1998).

[18] V. N. Baier and V. M. Katkov, Variation of radiation length due to LPM effect, Phys. Lett. A 327, 202 (2004).
[19] V. N. Baier and V. M. Katkov, Coherent scattering of high energy photon in a medium, Phys. Rev. D 63, 116008 (2001).

[20] U. A. Wiedemann and M. Gyulassy, Transverse momentum dependence of the Landau Pomeranchuk Migdal effect, Nucl. Phys. B560, 345 (1999).

[21] S. Klein, Suppression of bremsstrahlung and pair production due to environmental factors, Rev. Mod. Phys. 71, 1501 (1999).

[22] V. N. Baier and V. M. Katkov, Concept of formation length in radiation theory, Phys. Rep. 409, 261 (2005).

[23] P. L. Anthony et al., Bremsstrahlung suppression due to the Landau Pomeranchuk Migdal and dielectric effects in a variety of materials, Phys. Rev. D 56, 1373 (1997).

[24] H. Bethe and W. Heitler, On the stopping of fast particles and on the creation of positive electrons, Proc. R. Soc. London, Ser. A 146, 83 (1934).

[25] S. Weinberg, Infrared photons and gravitons, Phys. Rev. 140, B516 (1965).

[26] F. Bloch and A. Nordsieck, Note on the radiation field of the electron, Phys. Rev. 52, 54 (1937).

[27] J. D. Jackson, Classical electrodynamics, 3rd ed. (Wiley, New York, 1998).

[28] S. Weinberg, The Quantum Theory of Fields. Vol. 1: Foundations (Cambridge University Press, Cambridge, England, 1995).

[29] F. F. Ternovskii, On the theory of radiative processes in piecewise homogeneous media, Sov. Phys. JETP 12, 123 (1961).

[30] N. F. Shul'ga and S. P. Fomin, Effect of multiple scattering on the emission of ultrarelativistic electrons in a thin layer of matter, JETP Lett. 86, 32 (1998).

[31] R. J. Glauber, High-energy collision theory, Lect. Theor. Phys. 1, 315 (1959).

[32] X. Feal and R. A. Vazquez (to be published).

[33] V. L. Ginzburg and I. M. Frank, Radiation of a uniformly moving electron due to its transition from one medium into another, Zh. Eksp. Teor. Fiz. 16, 15 (1946).

[34] P. L. Anthony et al., Measurement of Dielectric Suppression of Bremsstrahlung, Phys. Rev. Lett. 76, 3550 (1996).

[35] P. L. Anthony et al., An Accurate Measurement of the Landau-Pomeranchuk Migdal Effect, Phys. Rev. Lett. 75, 1949 (1995).

[36] Y. Tsai, Pair production and bremsstrahlung of charged leptons, Rev. Mod. Phys. 46, 815 (1974). 